\title{
Conceptual design of the TPF-O SC buses
}

\author{
Lloyd R. Purves* \\ Code 592, NASA GSFC, Greenbelt, MD, USA
}

\begin{abstract}
The Terrestrial Planet Finder - Occulter (TPF-O) mission has two Spacecraft (SC) buses, one for a space telescope and the other for a formation-flying occulter. SC buses typically supply the utilities (support structures, propulsion, attitude control, power, communications, etc) required by the payloads. Unique requirements for the occulter SC bus are to provide the large delta $\mathrm{V}$ required for the slewing maneuvers of the occulter, and communications for formation flying. The TPF-O telescope SC bus shares some key features of the one for the Hubble Space Telescope (HST): both support space telescopes designed to observe in the visible to near infrared range of wavelengths with comparable primary mirror apertures (2.4 m for HST, $2.4-4.0 \mathrm{~m}$ for TPF-O). However, TPF-O is expected to have a Wide Field Camera (WFC) with a Field of View (FOV) much larger than that of HST. This WFC is also expected to provide fine guidance. TPF-O is designed to operate in an orbit around the Sun-Earth Lagrange 2 (SEL2) point. The longer communications range to SEL2 and the large science FOV require higher performance communications than HST. Maintaining a SEL2 orbit requires TPF-O, unlike HST, to have a propulsion system. The velocity required for reaching SEL2 and the limited capabilities of affordable launch vehicles require both TPF-O elements to have compact, low-mass designs. Finally, it is possible that TPF-O may utilize a modular design derived from that of HST to allow servicing in the SEL2 orbit.
\end{abstract}

Keywords: Terrestrial Planet Finder, TPF-O, occulter, space telescope, spacecraft bus

\section{INTRODUCTION}

Broadly speaking, a Terrestrial Planet (TP) is an exo-planet having approximately the mass of Earth and orbiting a sunlike star at distance of about 1 AU. Such a planet should be capable of sustaining life, and therefore has an expectation for showing signs of either life or a life-supporting environment. The detection and characterization of TPs are currently important astronomical goals. Thus, an important engineering question is how to design an efficient system to accomplish these goals. While the greatest technical challenges probably lie in the TPF-O payloads (namely the occulter, telescope, and instrument), the SC buses are nonetheless a key architectural element. TPF-O actually includes two SC buses (Occulter and Telescope), which need to be defined together because of the ways in which they interact. However, given the early stage of TPF-O definition, many of the SC Bus requirements and features are still qualitative in nature.

Because of the variety of functions performed by a SC bus, as summarized in Table 1, the overall TPF-O mission needs to be analyzed from a systems perspective to define the SC buses. This mission analysis starts with its basic objectives, which in the case of TPF-O, is the detection and characterization of TPs.

Because no existing telescope has the ability to identify even the potentially most observable TP, no such planets have yet been detected. This requires that TPF-O have an acceptably high probability of finding a $\mathrm{TP}$ based on an informed estimate of the frequency of these planets. Heap ${ }^{1}$ and Linder ${ }^{2}$ estimate that a $95 \%$ probability of detecting and characterizing a TP is achievable by a system that can:

Table 1. Typical Spacecraft Bus Functions

\begin{tabular}{|c|l|}
\hline 1 & Structural Support and Vibration Isolation \\
\hline 2 & Deployment and Pointing Mechanisms \\
\hline 3 & Temperature Sensing and Control \\
\hline 4 & Propulsion \\
\hline 5 & Attitude Sensing and Control \\
\hline 6 & Power Generation, Storage, and Distribution \\
\hline 7 & Communications for Commands, Telemetry and Tracking \\
\hline 8 & Command and Data Handling, including Flight Software \\
\hline
\end{tabular}

1 Structural Support and Vibration Isolation

2 Deployment and Pointing Mechanisms

4 Propulsion

6 Power Generation, Storage, and Distribution

7 Communications for Commands, Telemetry and Trackin

$$
\text { an: }
$$


1) For mirror apertures ranging from 2.4 to 4.0 $\mathrm{m}$, survey 44 to 101 of the most promising stars to detect TPs. This will require performing 102 to 134 surveys because of the probability that a planet will not be visible due to its location in its orbit at the time of a given survey.

2) Obtain $\mathrm{a} \sim 0.5$ to 1.1 micron spectrum with a spectral resolution of $\sim 70$ and a Signal-toNoise Ratio (SNR) $>=10$ from each detected TP to determine whether it has a life supporting environment. Larger apertures will provide a higher SNR. If the TP has such an Earth-like environment, then its measured spectrum could look like Figure 1.

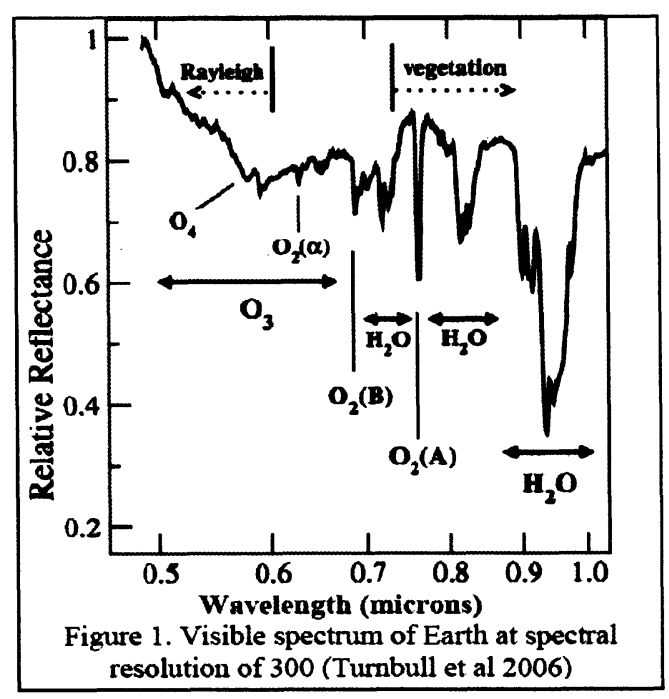

\section{TPF-O MISSION OVERVIEW}

The above performance requirements are taken as the primary ones for the TPF-O Mission, which is based on the concept of a visible/near-infrared (VIS/NIR) space telescope in an orbit about the Sun-Earth Lagrange 2 (SEL2) point that could be like the one in Figure 2. TPF-O uses an external, formation-flying occulter with a shape shown in Figure 3.

Figure 2. Potential TPF-O Orbit about Sun-Earth L2 Point

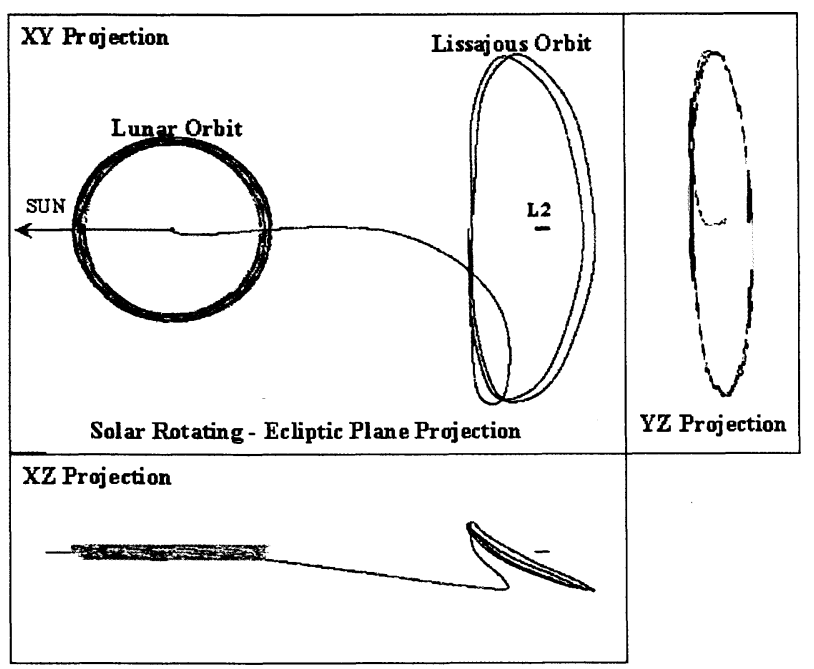

Figure 3. Occulter shape as seen by telescope

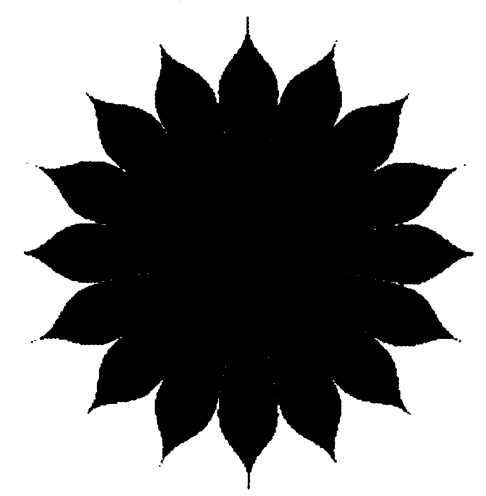

Using the alignment shown in Figure 4, This occulter shape is estimated to sufficiently suppress (factor of ten billion) the light of the TP's parent star. The other major TPF-O requirement is to be able to perform significant astrophysical observations during the substantial periods when the formation flying occulter is moving between the widely separated locations required for examining planets orbiting different target stars. 
Figure 4. Basic TPF-O Mission Architecture

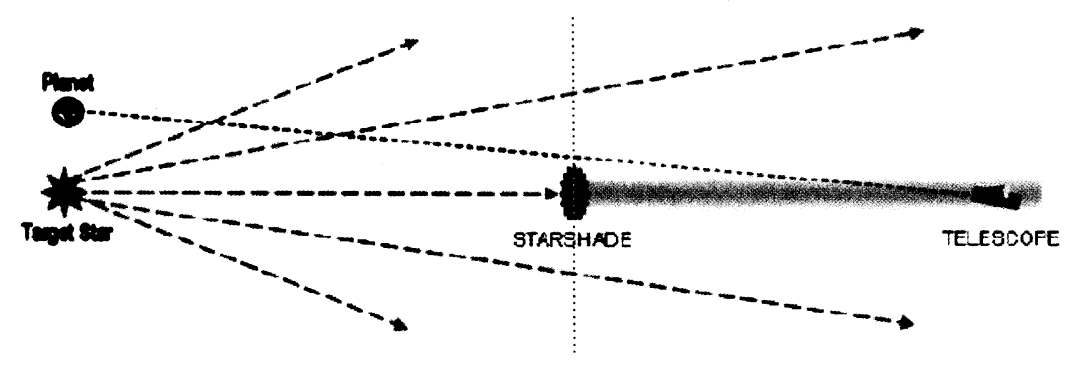

Table 2 summarizes the TFP-O architecture parameters to be analyzed. Given the unknown frequency of TPs and the difficulty of suppressing the light of the parent star to one part in 10 billion, it needs to be emphasized that this TPF-O architecture is far from final. Nonetheless, a systematic investigation of the $\mathrm{SC}$ bus requirements can yield information important for implementing the TPF-O mission.

Having specified the basic TPF-O architecture and orbit, the next

\begin{tabular}{|c|c|}
\hline & Table 2. Major TPF-O Architectural Parameters \\
\hline \multirow[t]{4}{*}{1} & $\begin{array}{l}2.4 \text { to } 4 \mathrm{~m} \text { aperture VIS/NIR telescope in orbit about the Sun- } \\
\text { Earth Lagrange } 2 \text { point with: }\end{array}$ \\
\hline & Planetary camera to detect Earth-like planets \\
\hline & Imaging spectrometer to characterize any detected planets \\
\hline & Wide field camera and spectrograph for astrophysics \\
\hline \multirow[t]{4}{*}{2} & Formation flying occulter with: \\
\hline & 16 petals \\
\hline & $\begin{array}{l}36.7 \text { (for } 2.4 \mathrm{~m} \text { aperture) to } 50 \mathrm{~m} \text { (for } 4.0 \mathrm{~m} \text { aperture) tip-to- } \\
\text { tip diameter }\end{array}$ \\
\hline & $\begin{array}{l}38,700 \mathrm{~km} \text { (for } 2.4 \mathrm{~m} \text { aperture) to } 72,000 \mathrm{~km} \text { (for } 4.0 \mathrm{~m} \\
\text { aperture) separation distance from telescope }\end{array}$ \\
\hline
\end{tabular}
question is the mission lifetime. This was chosen to be 5 years of science observations because it represents a reasonable maximum that can avoid the additional costs of full redundancy, highest parts quality, and more extensive testing. Lindler $^{2}$ developed a Design Reference Mission (DRM) simulation to determine how the TPF-O system could accomplish the science goals. Note that the DRM assumes that neither the position nor even the existence of a TP about a given star is known. A TP may exist but be unobservable due to its position being in front of or behind its star. The DRM may therefore observe a star more than once in order to see if a potential TP has moved to an observable position. The results of the DRM are summarized in Table 3, which indicates that 5 years provides the time for enough searches to obtain the spectra of 3 TPs.

\begin{tabular}{|l|c|c|}
\hline \multicolumn{3}{|c|}{ Table 3. TPF-O DRM Summary } \\
\hline DRM Parameter & $2.4 \mathrm{~m}$ Aperture & $4.0 \mathrm{~m}$ Aperture \\
\hline Total Period (days) & 1840 & 1832 \\
\hline Stars visited 1 time & 11 & 71 \\
\hline Stars visited 2 times & 15 & 27 \\
\hline Stars visited 3 times & 11 & 3 \\
\hline Stars visited 4 times & 7 & 0 \\
\hline Total number of stars observed & 44 & 101 \\
\hline Total number of visits & 102 & 134 \\
\hline Average Slew Distance (degrees) & 26.7 & 15.7 \\
\hline Slew Time: Average (days)/Total (\%) & $14.8 / 82.0$ & $10.99 / 80.4$ \\
\hline Observational Time: Average (days)/Total (\%) & $3.24 / 18.0$ & $2.67 / 19.6$ \\
\hline "Earths" Found (SNR of Spectra @ 0.94 micron) & $3(13,13,4.6)$ & $3(150,37,11)$ \\
\hline
\end{tabular}




\section{LAUNCH VEHICLE (LV) CONSIDERATIONS}

Although it is still too early to make a TPF-O LV decision, it is important now to understand LV limits and whether the occulter and telescope can be launched on one LV or two. To provide a sense of the limits, Figure 5 shows the largest available single and dual payload fairings. The US Delta IV Heavy launch vehicle, which can also send the heaviest payload mass $(\sim 9,500 \mathrm{~kg})$ to a SEL2 orbit, provides $\sim 4.6 \mathrm{~m}$ of payload diameter with cylindrical and total payload lengths of $\sim 11.0$ and $\sim 15.7 \mathrm{~m}$, respectively. The largest dual-payload fairing available is the ESA Ariane $\mathrm{V}$ fairing shown on the right, which provides the same $\sim 4.6 \mathrm{~m}$ payload diameter and overall payload lengths of $\sim 6$ and $\sim 10 \mathrm{~m}$. Note that the additional mass of a dual payload fairing can reduce the net payload mass by up to $1,000 \mathrm{~kg}$.

Figure 6. Concept of TPF-O Telescope mounted on occulter for launch (Courtesy of NGC)

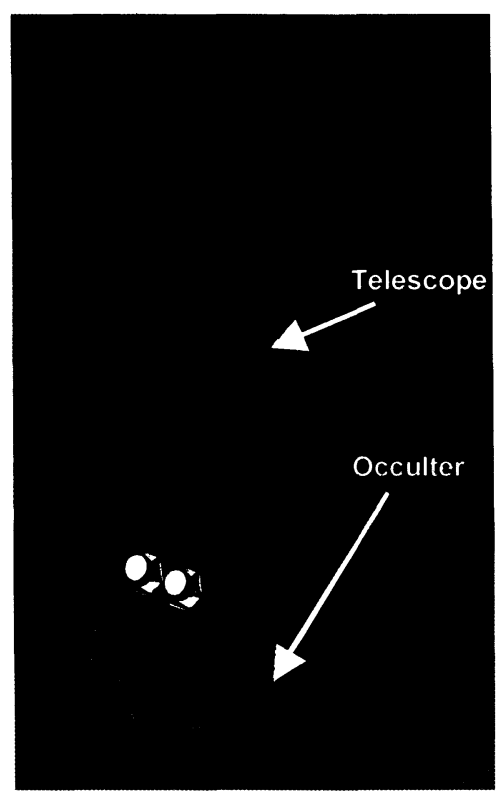

Figure 5. Largest Single and Dual Payload Fairings

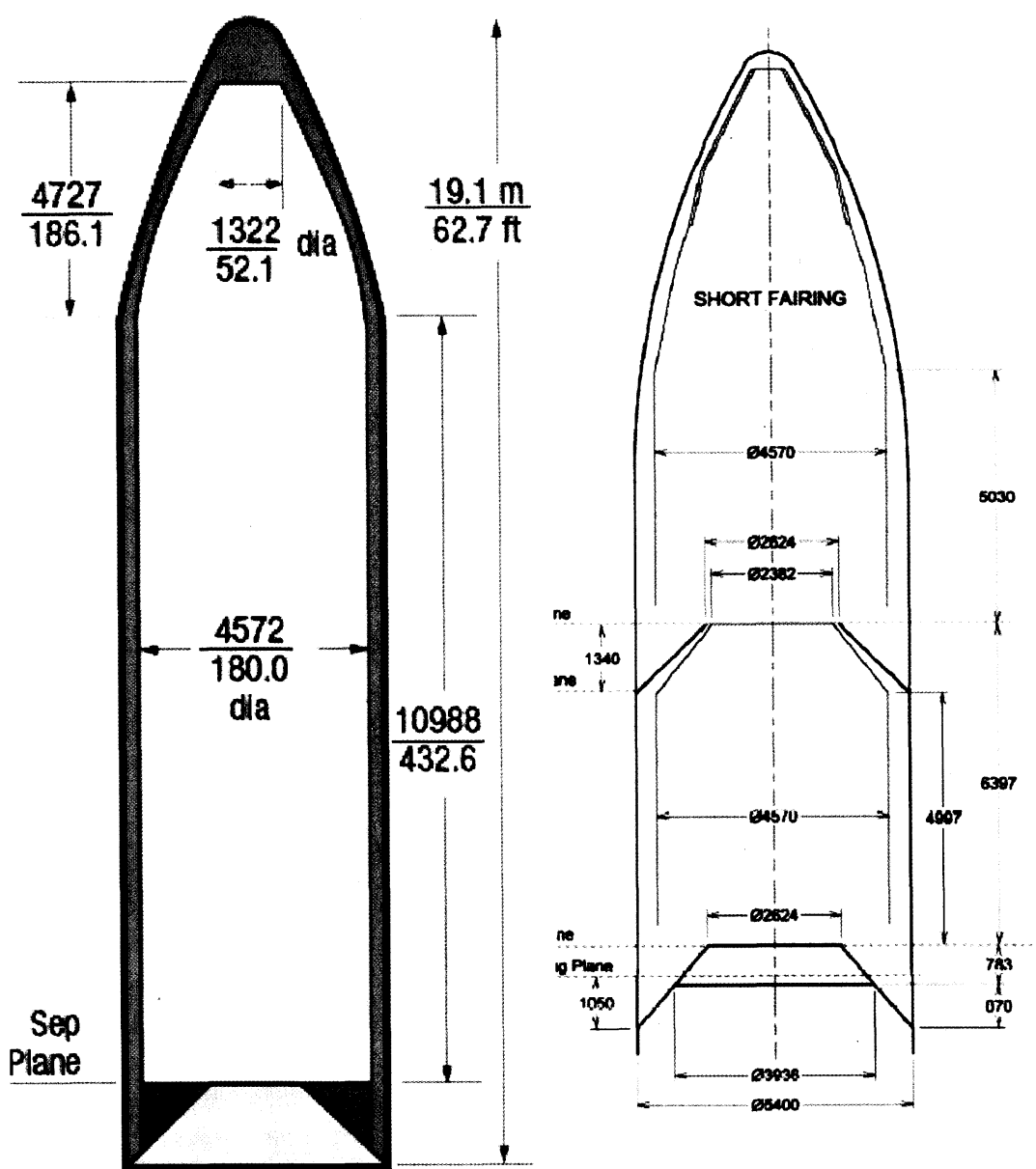

Delta IV heavy Composite Fairing

Ariane V Long SPELTRA

Another option, which is illustrated in Figure 6, is to strengthen the occulter structure enough for it to carry the telescope in the launch vehicle. However, this additional occulter mass will also require more propellant for occulter maneuvers, so this option needs careful analysis. 


\section{OCCULTER SPACECRAFT BUS}

The occulter SC bus will be treated first because it needs to provide critical formation flying signals that the telescope SC bus must accommodate. As shown in Figure 7, the occulter SC bus does not include the occulter itself, which represents the payload and is for the most part a very specialized deployable structure. Some qualitative and quantitative considerations for the major SC bus subsystems are summarized below.

Occulter Shade Interface: The interface between the SC bus and occulter shade will include mechanical support, power for the deployment and thermal control of the shade, bi-directional data for deployment and thermal control commands, plus housekeeping telemetry.

Structure: The basic structural functions will be to tie together all of the occulter components, interface to the launch vehicle, and provide the stiffness required for the launch and operational phases. The structure is also expected to include damping that will sufficiently isolate the occulter shade from the vibration produced by the ACS and propulsion systems.

Mechanisms: One primary function identified is to deploy and point the large solar arrays. Another is to gimbal the propulsion thrusters so that the thrust vector passes through the Figure 7. External Features of Occulter SC Bus

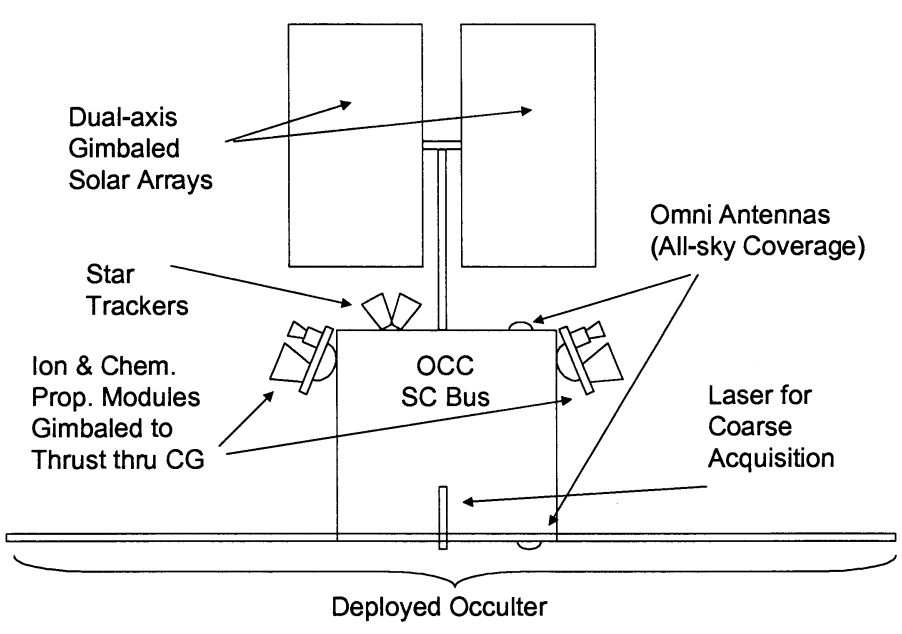

Occulter Center of Gravity (CG), which will shift as propellant is depleted.

Thermal Control System: This is expected to consist of normal heaters, thermocouples, and radiators required to maintain the SC Bus equipment within acceptable temperature ranges.

Propulsion: Due to the extremely tight station keeping required during TP observations and the very high delta V required to move the occulter when the telescope slews between target stars, two forms of propulsion appear to be required. Chemical propulsion will maintain the occulter on target, and ion propulsion will be used for traveling between targets. Figure 8 shows the chemical propulsion concept for keeping the center of the occulter within $\sim 1 \mathrm{~m}$ of the LOS between the telescope and the target star. Given the low differential gravitational acceleration (about $1 \mathrm{E}-5 \mathrm{~m} / \mathrm{sec}^{2}$ ) between the occulter and telescope, very short chemical thrusters firings will occur at intervals of more than 1,000 seconds.

Between targets, the occulter has to move an average distance relative to the telescope that is twice its distance from the telescope times the sine of half of the telescope slew angle. Based on the average slew angle shown in Table 3 for the $4.0 \mathrm{~m}$ aperture, this is an average relative move of $\sim 20,000 \mathrm{~km}$ taking an average time of $\sim 11$ days,

Figure 8. Occulter Propulsion in Observation Mode

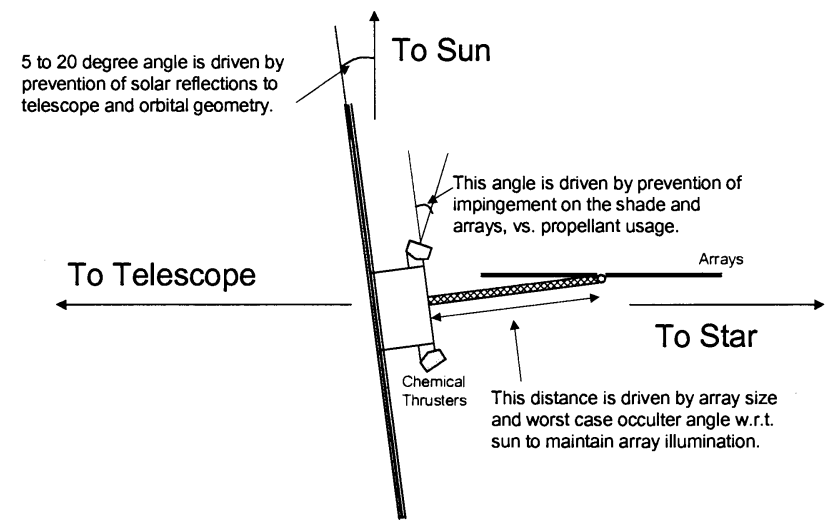

$.230 \mathrm{~m} / \mathrm{s}$ ? $\mathrm{V}$ required for chemical position maintenance 
and performed 134 times.

However, both the telescope and occulter are always orbiting around the SEL2 point, as shown in Figure 9, so guiding the occulter slews is complex. Figure 10 shows the ion propulsion concept for the occulter and presents some propulsion sizing estimates for an earlier DRM that is similar, but not identical, to those in Table 3. Note that a total of $\sim 10 \mathrm{~km} / \mathrm{sec}$ of Delta $\mathrm{V}$ is required.

Figure 9. Occulter (Blue for Observation and Green for Slew) and Telescope (White) Trajectories

Another component of the propulsion system is expected to be a very sensitive (possibly proof-mass) accelerometer. By allowing the occulter to accurately measure acceleration, the accelerometer can improve onboard estimates of velocity and position enough to allow a significant degree of autonomous navigation.

Figure 10. Occulter Ion Propoulsion Concept

(Courtesy of GRC)

- Reference TPF-O Mission

- Transfer maneuvers modeled as free-space

- Position maintenance modeled as a fixed acceleration of $1 \times 10^{-5} \mathrm{~m} / \mathrm{s}^{2}$

- 63,000 km from telescope to occulter

- 24.6[average telescope slew between observations

- 5 yr duration ( 124 targets with avg. of 2.2 day for obs \& 12.6 day for transfer)

- $10.8 \mathrm{~km} / \mathrm{sec}$ Total occulter? V

- Reference Occulter Configuration

- $2500 \mathrm{~kg}$ wet spacecraft

- $600 \mathrm{~kg}$ of Xenon Propellant

- 2 NEXT ion thrusters on each of 2 opposing spacecraft sides

- One nominal, one back-up for each side

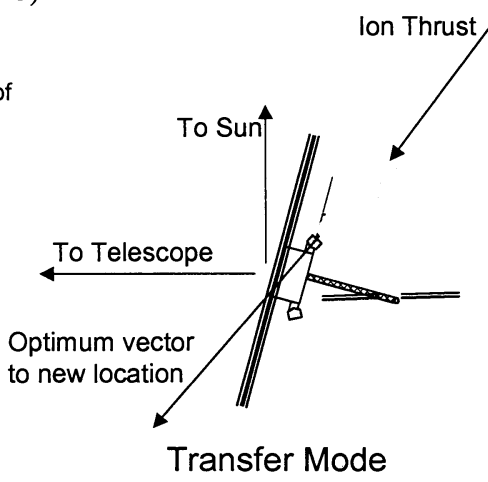

- $6.9 \mathrm{~kW}$ per thruster

- $236 \mathrm{mN}$ per ion thruster

- $4170 \mathrm{sec}$ Isp 
Attitude and Control: Although the occulter places challenging position control requirements on the propulsion system, its pointing control requirements are relatively modest. Basically, a normal complement of reaction wheels for 3 -axis control, plus star trackers and gyros should be adequate. Note that the gimbaling of the thrusters so that the thrust vector goes through the CG is part of the attitude control.

Power: The major requirement on the power system will be solar arrays to generate on the order of $10 \mathrm{~kW}$ of power that is mainly required by the ion thrusters. Because a SEL2 orbit is only about $1 \%$ further from the sun than the Earth, the solar arrays will function with the same efficiency as they do near Earth. The mast and two axis gimbals shown in the Figures 7 should be sufficient to keep the arrays in full sunlight and pointed to the sun during any anticipated maneuvers.

Communications: Because the occulter does not produce science data, it does not require high bandwidth communications. It will require bi-directional communications with ground stations to receive commands (mostly for its slew maneuvers) and to transmit housekeeping telemetry. It will also require a ranging transponder to support ground tracking for guidance during its slew maneuvers, although some or all of this could in principle be accomplished with appropriate on-board sensors (cameras and accelerometers).

In addition, as described by Leitner ${ }^{3}$, formation flying imposes extra communications requirements on the occulter. Using ground tracking data (as described above), the occulter will be only coarsely positioned (tens of kilometers) relative to its required location when it reaches the end of a slew maneuver. At that point the occulter will communicate with the telescope by flashing a laser. A telescope science camera will detect the laser flashes and provide the data to determine where the laser is relative to the target star. Based on this information, the occulter will be commanded to move to within about $10 \mathrm{~m}$ of its required position. With this positional accuracy, the occulter will begin blocking the light that the telescope sees from the target star. To accomplish this, the occulter must be able to receive data from the telescope, possibly simultaneously with ground communications. The RF data received from the telescope should also pass through a ranging transponder so that the occulter-telescope separation distance can be calculated and controlled.

Lyon ${ }^{4}$ describes how, when the occulter is blocking starlight, a telescope pupil camera and quad cell detector can determine the offset between the center of the occulter and the LOS from the telescope to the target star. This measurement of the diffracted longer wavelength starlight can be made accurately enough to generate the commands for the occulter to move its center to within $\sim 1 \mathrm{~m}$ of the LOS and maintain it. These measurements will be communicated from the telescope to the occulter in the same fashion as those for the laser.

Probably all of the occulter RF communications can be S-band. All sky viewing Omni antennas will certainly be required for contingencies. Whether MGA or HGA antennas will also be required needs more study, but none of the occulter RF or laser communications requirements presently look challenging.

Command \& Data Handling/Flight Software: No special requirements are seen at this point in time. However, the occulter could in principle make good use of sophisticated on-board orbit determination algorithms. With the required sensors, this software could allow fairly autonomous navigation during the long slew maneuvers, and may be able to reduce the number of station keeping maneuvers by applying predictive techniques.

\section{TELESCOPE SC BUS}

Figure 11 shows the Telescope SC bus in relationship to the telescope and instruments. In a basic way, the architecture of the TPF-O telescope and bus can be likened to that of HST, with the main difference being that the TPF-O telescope will require the addition of a propulsion system to get into and maintain its SEL2 orbit. By comparison, HST uses the Shuttle's propulsion for periodic orbit raisings. Requirements for the SC bus subsystems are summarized below.

Telescope and Instrument Interface: A challenging interface between the SC bus and the telescope payload is expected to be the mechanical support. This is because of the need to isolate the telescope from vibration sources, such as the ACS. Active isolation may be required, depending on the telescope performance specifications.

Other interfaces will include power for telescope avionics and thermal control, a low relatively low speed bi-directional link (possibly 1553) for instrument commands and housekeeping telemetry, plus a high-speed link (possibly multiple parallel links) to bring the science data to the solid-state recorder (SSR) on the SC bus. Until the complement of 
instruments and detectors is better defined, the required bandwidth of the high-speed link cannot be estimated. However, given the feasibility of parallel links, there is no reason to assume that any technology development will be required.

Figure 11. Telescope SC Bus (External Features)

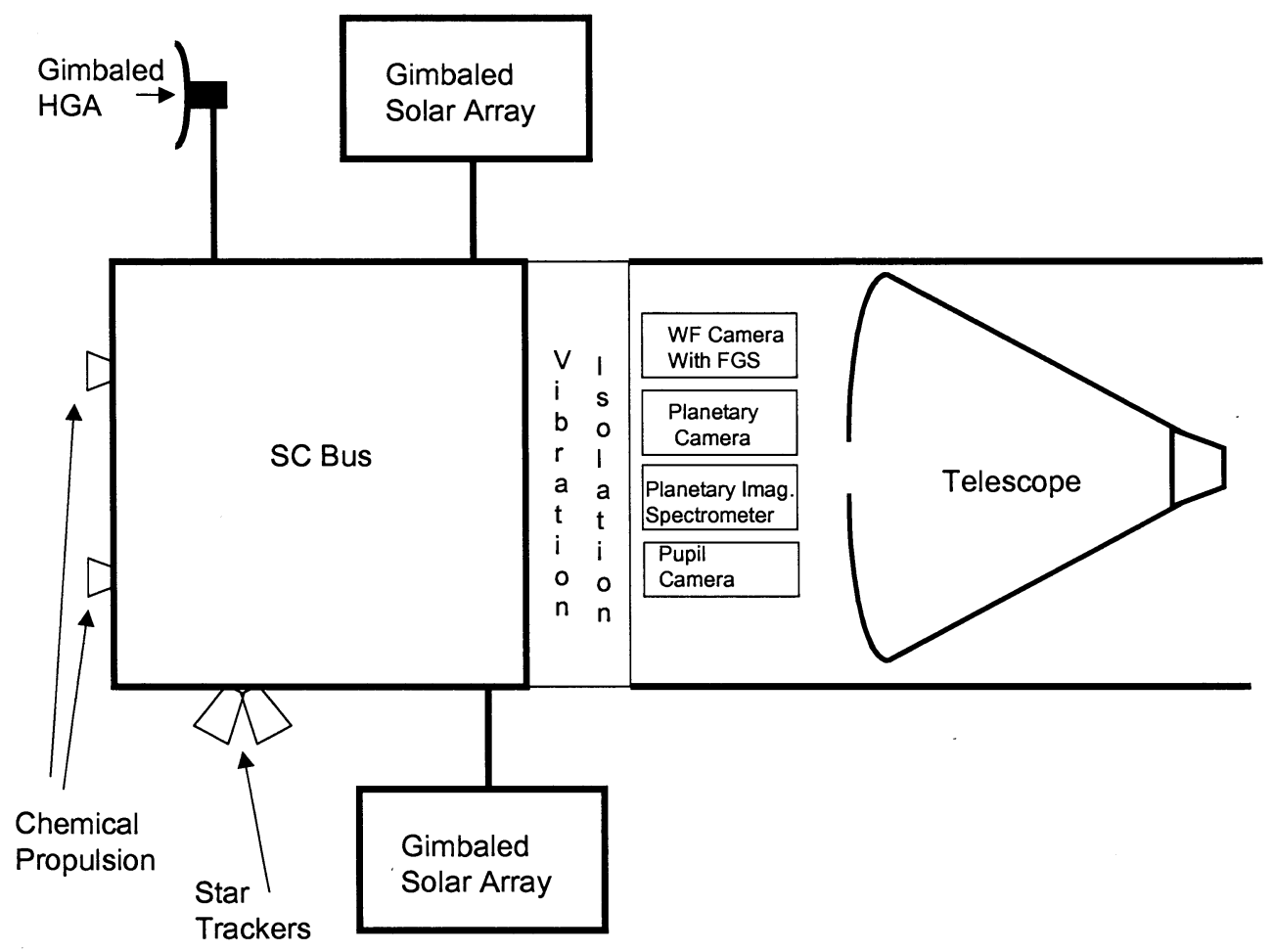

Structure: As with the occulter, the basic structural functions will be to tie together all of the components, interface to the launch vehicle, and provide the stiffness required for the launch and operational phases. The structure may also incorporate multiple levels of vibration isolation, such as dedicated dampers for the reaction wheels and passive or active damping between the SC bus and the telescope/instrument payload.

Mechanisms: The primary functions identified will be to deploy and point both the solar array and the High Gain Antenna (HGA).

Thermal Control System: Like the occulter, this is expected to consist of normal heaters, thermocouples, and radiators required to maintain the SC Bus equipment within acceptable temperature ranges. However, if the telescope uses lowtemperature detectors, then a cryo-cooler may also be required.

Propulsion: The primary requirements on the propulsion system will be to: correct launch vehicle dispersions, provide midcourse correction burns on the transfer trajectory to the SEL2 orbit, perform the orbit insertion burn, and provide small station keeping burns every few week maintain the SEL2 orbit. The propulsion system may also have to provide momentum-dumping burns. Even if the design lifetime of TPF-O is 5 years, it will statistically have a very good chance of providing useful science for much longer, so enough propellant may be included to allow up to a ten-year mission.

The requirements on the telescope propulsion system, on the order of a total of a few hundred meters per second, may be modest enough to be met with a relatively simple mono-prop system, similar to that on JWST. 
Attitude and Formation Flying Control: The telescope will require 3-axis attitude control. The basic ACS components are the normal ones of star-trackers, gyroscopes, reaction wheels, sun sensors for safe-hold modes, and the associated control algorithms, typically implemented in the flight software of the C\&DH subsystem.

However, the telescope payload will have to provide the sensing required for the fine-pointing requirements of the telescope. Currently, it is anticipated that the wide-field camera (WFC) will contain detectors having sufficient field of view, sensitivity and readout speed to supply the required fine-pointing knowledge. If there is an active vibration isolation system, this could in principle also provide the fine pointing control for the telescope.

Although more precisely described as formation flying control functions, the various cameras of the telescope will also feed observations of the occulter to the flight software that will estimate the position of the occulter relative to the line of sight between the telescope and a target star. Either the planetary or WF camera will detect the position of the occulter laser beam, from which the flight software will calculate how far the occulter must move to reach the line of sight to the target star. Upon reaching the line of sight, the pupil camera will detect the pattern of the diffracted longer wavelength light from the target star, and the flight software will determine what changes in the occulter position will be required to be stay within $\sim 1 \mathrm{~m}$ of the star's line of sight. In parallel, the telescope-to-occulter RF ranging system will measure the separation distance between the occulter and the telescope.

Power: Without the need to power ion engines, the solar arrays should only have to generate a total of a few $\mathrm{kW}$ of power. As with the occulter, the solar arrays will have the same efficiency as those near Earth. Fixed, body-mounted solar arrays could in principle prove adequate. However, for a variety of reasons (compact stowed configuration, freedom to hold indefinitely any orientation with respect to the sun, room for thermal radiators), it is expected that the solar arrays will be deployed and gimbaled. Battery requirements should be modest as the telescope can always be in full sunlight.

Communications: The telescope will require all sky viewing, bi-directional, S-band omni antennas for ground communications to support safe-hold operations and other possible contingencies. The telescope will also have a ranging transponder to support tracking using ground stations.

As noted earlier, the telescope needs to bi-directionally communicate with both the occulter and the ground, probably at the same time. The RF link to the occulter will be primary for tracking the occulter and issuing it repositioning commands. Gimbaled high gain antennas do not seem needed for this function, but analysis will have to determine whether the omni antennas will be sufficient or whether fixed medium gain antennas will also be needed.

The main communications challenge for the telescope could be that of down-linking the science data. While specifications have not yet been set for the TPF-O WFC, it could be designed to have well over a billion pixels on a 4-m telescope. Depending on the full well depth, the integration time, the amount of onboard data compression, and the cost of ground station contact time, the communication system could have very high performance requirements. The highest communications rate expected to be achievable in the TPF-O timeframe (without technology development) is $1 \mathrm{Gbps}$ using a large steer-able Ka-band HGA, powerful RF transmitters, and large-antenna ground stations.

It is technically possible for the three DSN stations to maintain near constant contact with a SEL2 observatory, but this is not programmatically realistic. Dedicated ground antennas could prove cost effective, but this needs to be studied.

Command \& Data Handling/Flight Software: No special requirements are seen at this point in time. A large Solid State Recorder (SSR) may be necessary depending on the total number of pixels, onboard data compression, and frequency of ground contact, however with Trillion bit (Tb) capacity SSRs available today, this requirement does not seem to pose any technical challenges. No significant new algorithm development appears required for the FSW.

\section{SERVICING}

The HST mission has repeatedly demonstrated the feasibility and value of servicing a space telescope using ExtraVehicular Activity (EVA). However, there are no current plans to make EVA servicing available in a SEL2 orbit. The time, risk, and propellant demands for first bringing TPF-O elements back to Low Earth Orbit (LEO) for EVA servicing 
and then returning them to SEL2 are prohibitive. While establishing a human presence at the moon could make EVA servicing of TPF-O feasible in principle, the option of robotic servicing is also technically possible.

The servicing of any space satellite consists for the most part of replacing any module (often called an Orbital Replacement Unit or ORU) that has developed problems or become obsolete. Remotely and autonomously controlled robotic servicing of space assets has long been considered technically feasible assuming that the ORUs are designed to be "robot-friendly", i.e. easy for a robot to autonomously track, grasp, detach and attach. Recently DARPA successfully demonstrated that approach in space on the Orbit Express mission, whose major features are summarized in Figure 12. Basically, it would appear that OE has provided a high Technology Readiness Level (TRL) for all of the tools and processes required to service TPF-O.

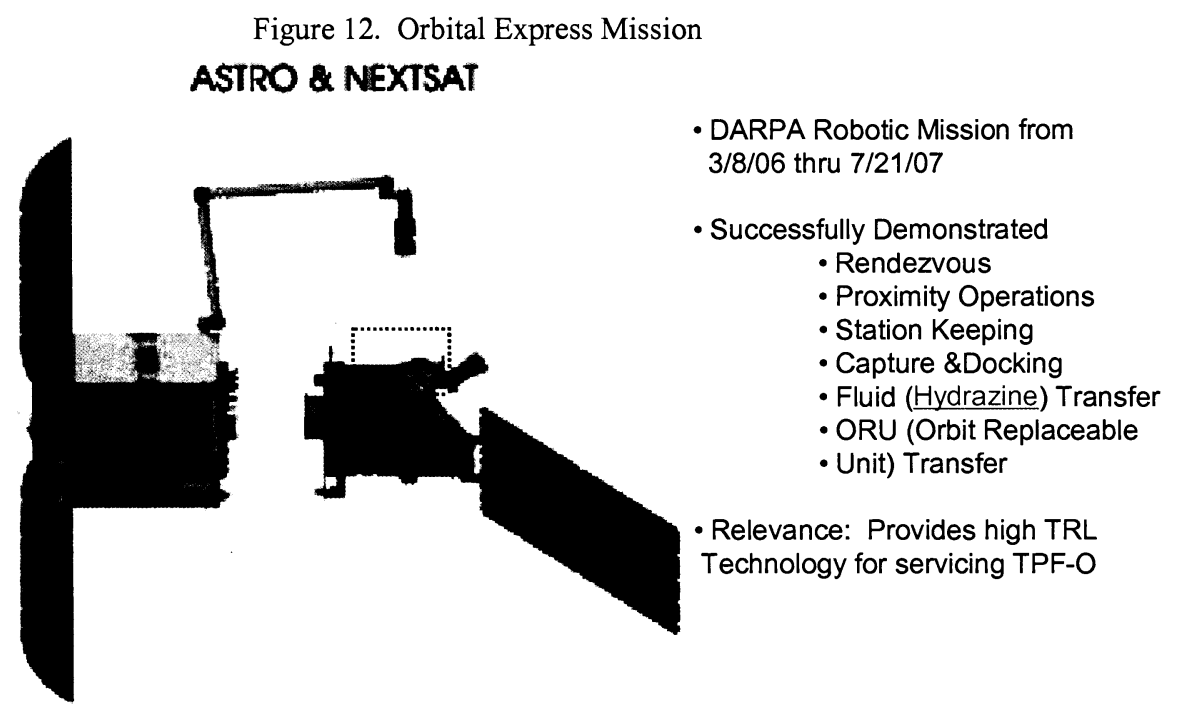

Robotic servicing of TPF-O in a SEL2 orbit could extend its lifetime and upgrade its capabilities, as EVA has done with HST. However, there are significant additional costs associated with robotic servicing, so careful study will be required to determine if it is worthwhile.

\section{CONCLUSIONS}

There is ample heritage for the environments and functions of the TPF-O SC buses, examples of which include:

- HST: Serviceable SC Bus for UV/VIS/NIR ST

- SOHO in SEL1 Halo Orbit: L2-like Halo Orbit dynamics

- WMAP in SEL2 Tight Lissajous Orbit: L2 Environment

- JWST: ST in SEL2 orbit being considered for TPF-O

- OE: Robotic servicing demonstration

No technical challenges are seen for the TPF-O SC buses that will require significant R\&D. The high delta $\mathrm{V}$ required for the occulter can be achieved with today's ion propulsion technology. The tight occulter formation-flying requirements will place demands on the SC bus payloads (stability of the occulter shade and tracking ability of the telescope), but not on the SC buses themselves.

However, TPF-O is still in its conceptual design phase and there are many SC bus-related trades that should be done. One trade covered above indicates that the same basic SC bus architecture can be used for telescope primary mirror apertures from 2.4 to $4.0 \mathrm{~m}$. Examples of other possible trades include: 
- Servicing options, such as keeping parts of the robot servicing system in SEL2 orbit for reuse, which causes them to also become candidates for servicing.

- Lunar-phasing trajectory to a SEL2 orbit (used by WMAP) to facilitate returning to lunar environment where EVA servicing might someday be available

- A Drift-away orbit could allow the telescope to dispense with its propulsion system

- Laser communications to compensate for the greater distances that TPF-O would be from Earth in a driftaway orbit

- Feasibility of fitting the occulter and telescope onto a single LV either by using a dual payload adapter or by having the occulter support the telescope during launch

- Upgrading the launch vehicle to reduce dispersions versus providing the propellant for TPF-O to compensate for dispersions

- On-board sensors and software to substantially reduce the need for ground tracking

- Dedicated (possible lower performance) ground stations versus shared, higher performance ones.

- Next generation ion engines (e.g., NEXT) versus current ones, such as NSTAR

- Smaller number of gimbaled thrusters versus more fixed thurster

\section{REFERENCES}

${ }^{1}$ S. Heap, “The Terrestrial Planet Finder-Occulter (TPF-O) Science Program”, SPIE, 6687-32 (2007)

${ }^{2}$ D. Lindler, "TPF-O design reference mission”, SPIE, 6687-33 (2007)

${ }^{3}$ J. Leitner, "Formation flying system design for a planet-finding telescope-occulter system", SPIE, 6687-39 (2007)

${ }^{4}$ R. Lyon, "Externally occulted terrestrial planet finder coronagraph: simulations and sensitivities", SPIE, 6687-37 (2007) 


\title{
Conceptual design of the TPF-O SC Buses
}

\author{
Lloyd Purves \\ NASA GSFC Code 592
}




\section{Introduction}

- Detection and characterization of Terrestrial Planets (TP) is currently an important astronomical goal

- Design of an effective TP Finder (TPF) mission is therefore an important engineering challenge

- Objective is initial look at design of SC buses for TPF-O Occulter and Telescope

- Multi-Functional Nature of SC bus (structure, mechanisms, thermal control, propulsion, attitude control, power, communications, command \& data handling) means that end-end systems analysis is required

- Need to start with Occulter bus functions to understand requirements on telescope bus 


\section{Background}

- Quarry (Terrestrial Planets):

- Orbits a sun-like star

- Located within habitable zone ( 1 AU from star)

- Approximately mass of Earth

- Frequency of Terrestrial Planets

- None yet observed

- No existing telescope has sufficient capability

- Estimated to occur around $10 \%$ of sun-like stars

- Probability Defined as Eta-sub-Earth $=0.1$ 


\section{TPF Mission Requirements}

- Needs to have a good ( $25 \%$ probability) of detecting and characterizing a TP

- Estimated to require:

- 230 surveys of habitable zones around sun-like starts within $10 \mathrm{PC}$

- 100 surveys will be required because (statistically) planets will not always be visible due to being in front of or behind star

- 60 different stars may be visited, some multiple times, because multiple visits to one star are equivalent to single visits to multiple stars

- Characterize planets detected in habitable zone

- Obtain 0.5 - 1.0 micron spectra of with spectral resolution of $\sim 70$ and $\mathrm{S} / \mathrm{N}$ of $>=10$

- Spectral features of interest: oxygen, water vapor, etc 


\section{TPF-O Mission Overview}

- Based on concept of using formation flying external occulter to suppress light of parent star by $1 \mathrm{E} 10$ (ten billion)

- Reduces parent-star light to level of light from TP

- Avoids scattered light problems of internal occulter

- External occulter has flower-like petals which suppress diffracted light

- $95 \%$ probability of finding a TP estimated to require

- $4 \mathrm{~m}$ aperture VIS/NIR telescope in SEL2 orbit for 5 years

- 16 petal, $50 \mathrm{~m}$ tip-tip diameter occulter, 72,000 km from telescope

- Result checked with simulated Design Reference Mission (DRM)

- Includes WF camera for astrophysical observations during the $\sim 80 \%$ of DRM time that occulter is repositioning itself between target star locations 


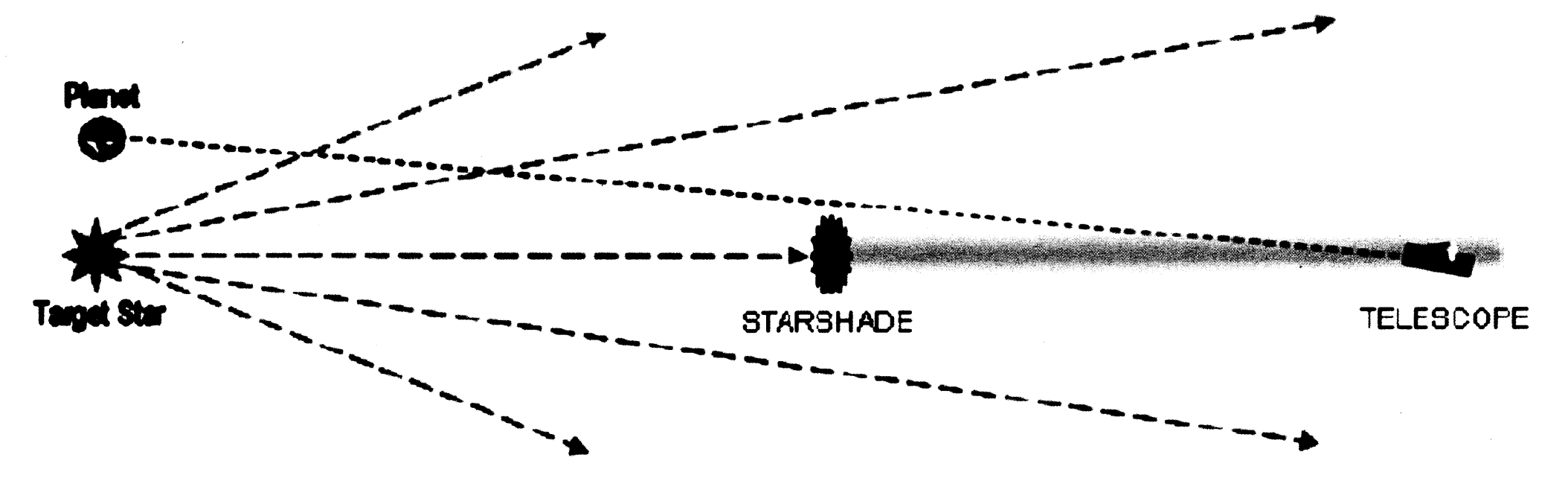

Figure 2. TPF-O throws a deep stellar shadow over the telescope, but allows planet light to pass by the starshade

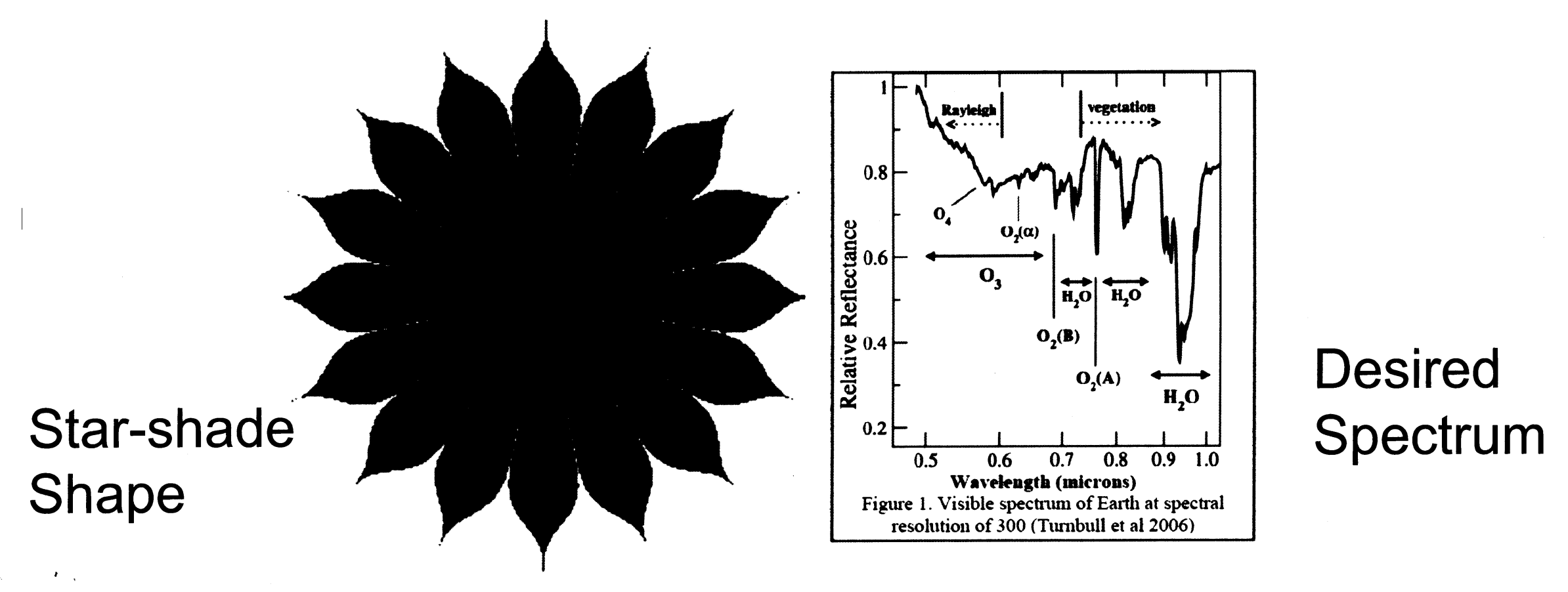




\section{TPF-O DRM}

- Total Period of DRM $=1832$ days

- Total number of visits $=134$

- $\quad$ Total number of stars observed $=101$

71 stars visited 1 time

27 stars visited 2 times

3 stars visited 3 times

- Average of 1.3 visits/star

- Average Slew Distance $=15.7$ degrees

- $\min \max$ avg. total percent

• $\quad$ -

- $\quad$ Time on target (days) $\quad 0.8139 .52 \quad 2.67 \quad 358.19 .56 \%$

- Time during slews (days) 4.4520 .8710 .99 1473. $80.44 \%$

- Predicted Results

Three "Earths" Found spectra taken with $S N=150, S N=37$, and $S N=11$

Spectra of 35 "Jupiters" taken. 


\section{Direct Injection to L2 Orbit}

(JWST Approach, 100 days from launch to Orbit

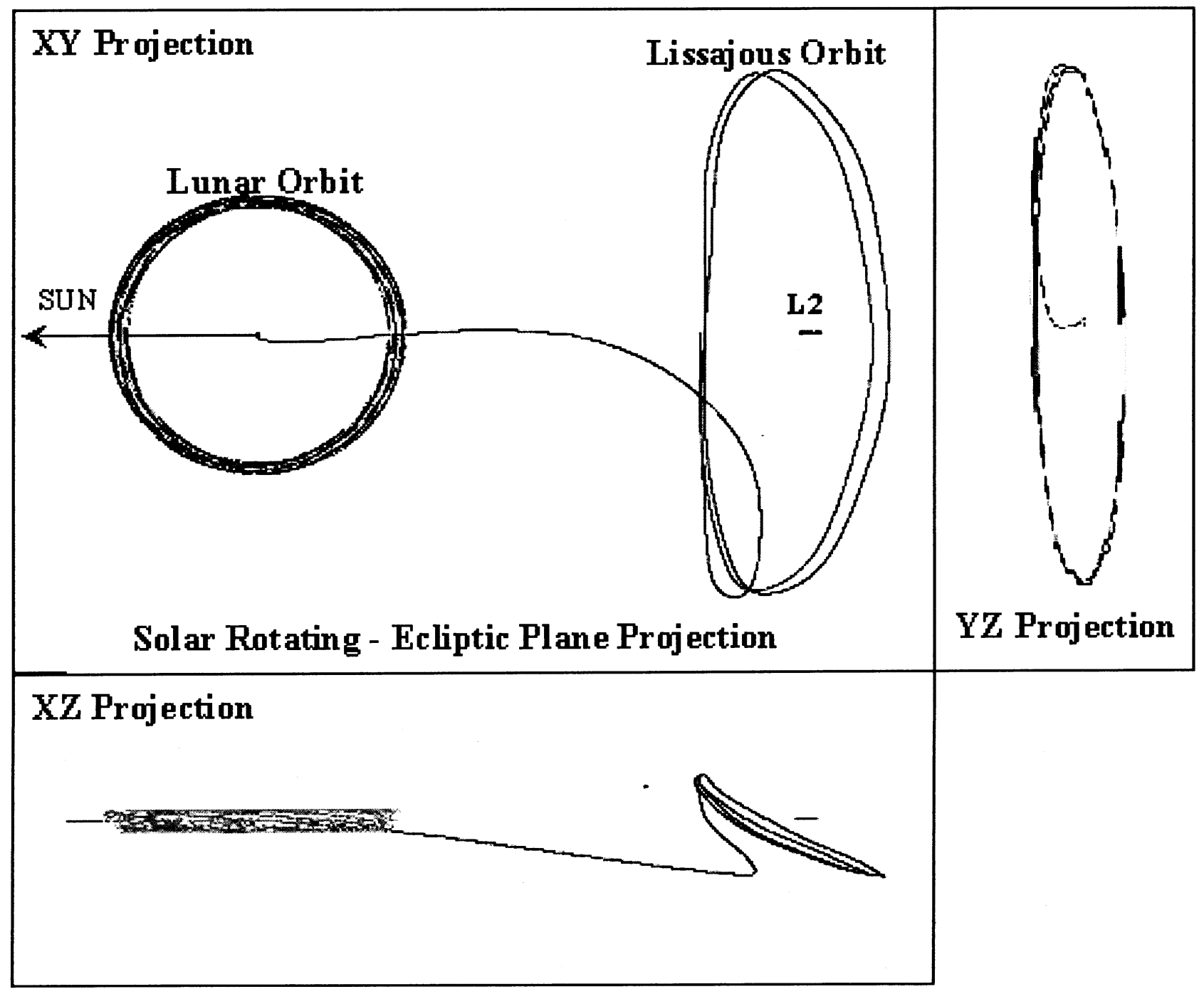




\section{Occulter Targeting Detail}




\section{Occulter in Observing Mode - Courtesy of GRC}

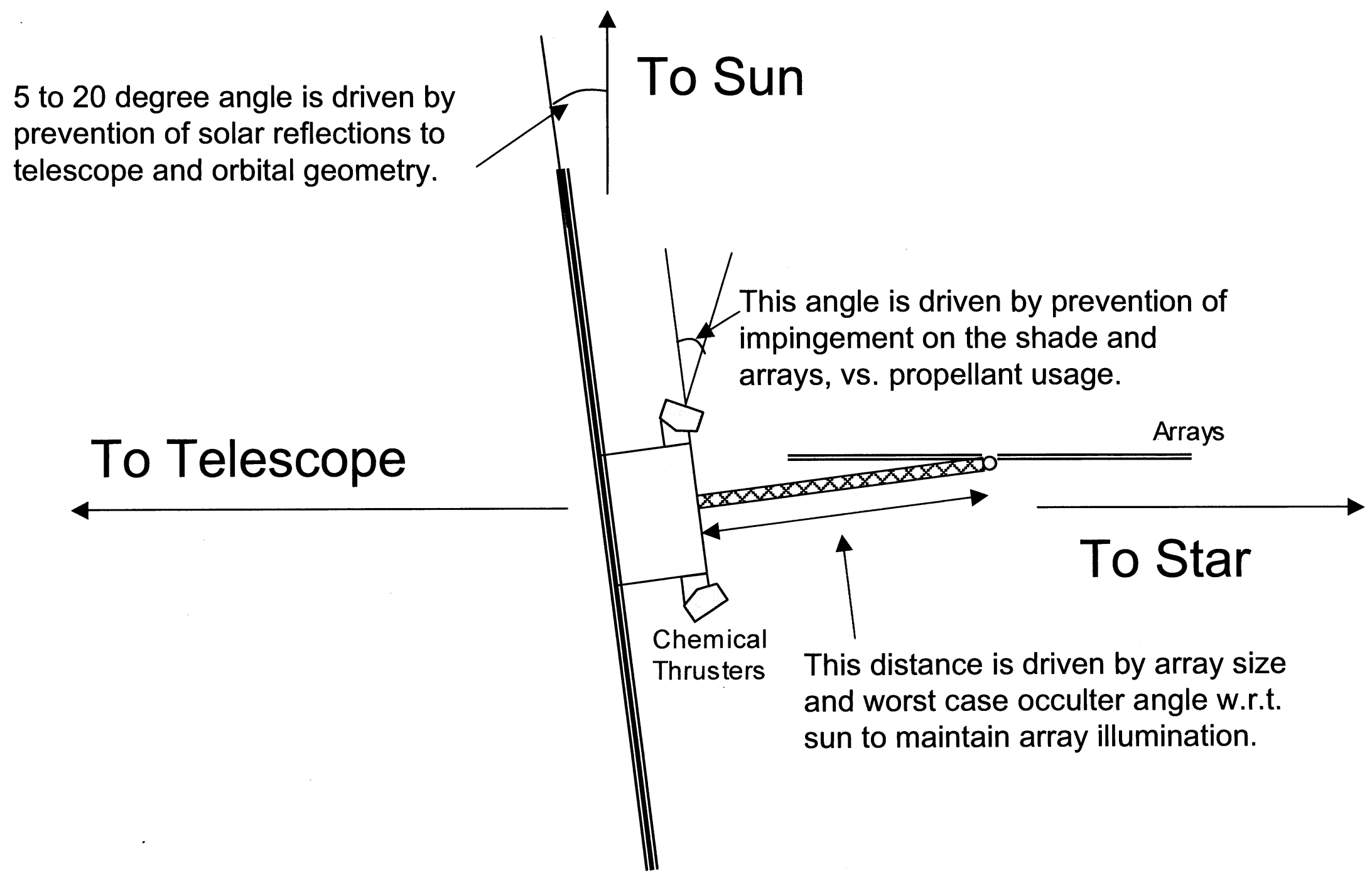

$.230 \mathrm{~m} / \mathrm{s} \Delta \mathrm{V}$ required for chemical position maintenance 


\section{Occulter in Transfer Mode - Courtesy of GRC}

- Reference TPF-O Mission

- Transfer maneuvers modeled as free-space

- Position maintenance modeled as a fixed acceleration of $1 \times 10^{-5} \mathrm{~m} / \mathrm{s}^{2}$

- 63,000 km from telescope to occulter

- $24.6^{\circ}$ average telescope slew between observations

- 5 yr duration (124 targets with avg. of 2.2 day for obs \& 12.6 day for transfer)

- $10.8 \mathrm{~km} / \mathrm{sec}$ Total occulter $\Delta V$

- Reference Occulter Configuration

- $2500 \mathrm{~kg}$ wet spacecraft

- $600 \mathrm{~kg}$ of Xenon Propellant

- 2 NEXT ion thrusters on each of 2 opposing spacecraft sides

- One nominal, one back-up for each side

- $6.9 \mathrm{~kW}$ per thruster

- $236 \mathrm{mN}$ per ion thruster

- 4170 sec Isp

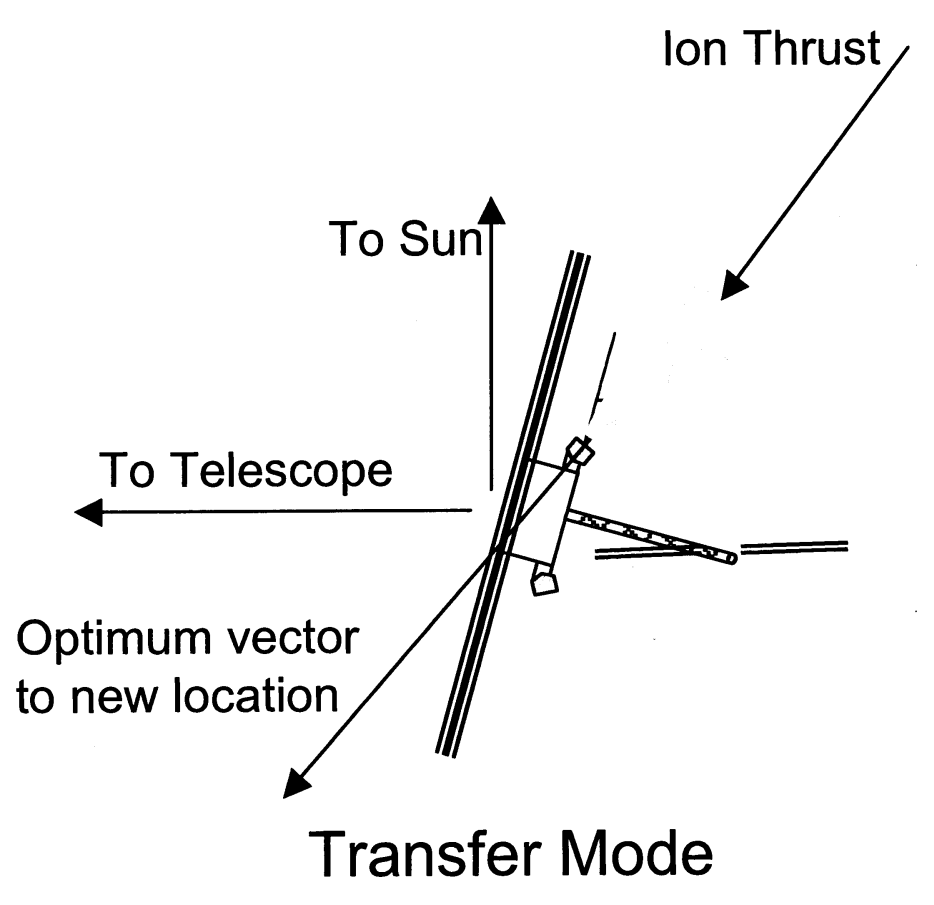




\section{Launch Vehicle Considerations}

- Delta IV Heavy Provides Largest Single Payload Capability to SEL2 Orbit

- Mass (kg): 9500

- Diameter $(\mathrm{m}): \sim 4.5$

- Length $(\mathrm{m}): \sim 12.2$ Cylindrical \& $\sim 16.4$ Total

- Other Options

- Use Ariane V Type Dual Payload Adapter

- Could impose up to $1,000 \mathrm{~kg}$ mass penalty

- Have Occulter carry Telescope in LV

- Requires Occulter to have more massive structure, whicu requires more propellant

- Conclusion

- Two launch vehicle approach looks feasible

- Occulter \& ST on single LV may be possible 


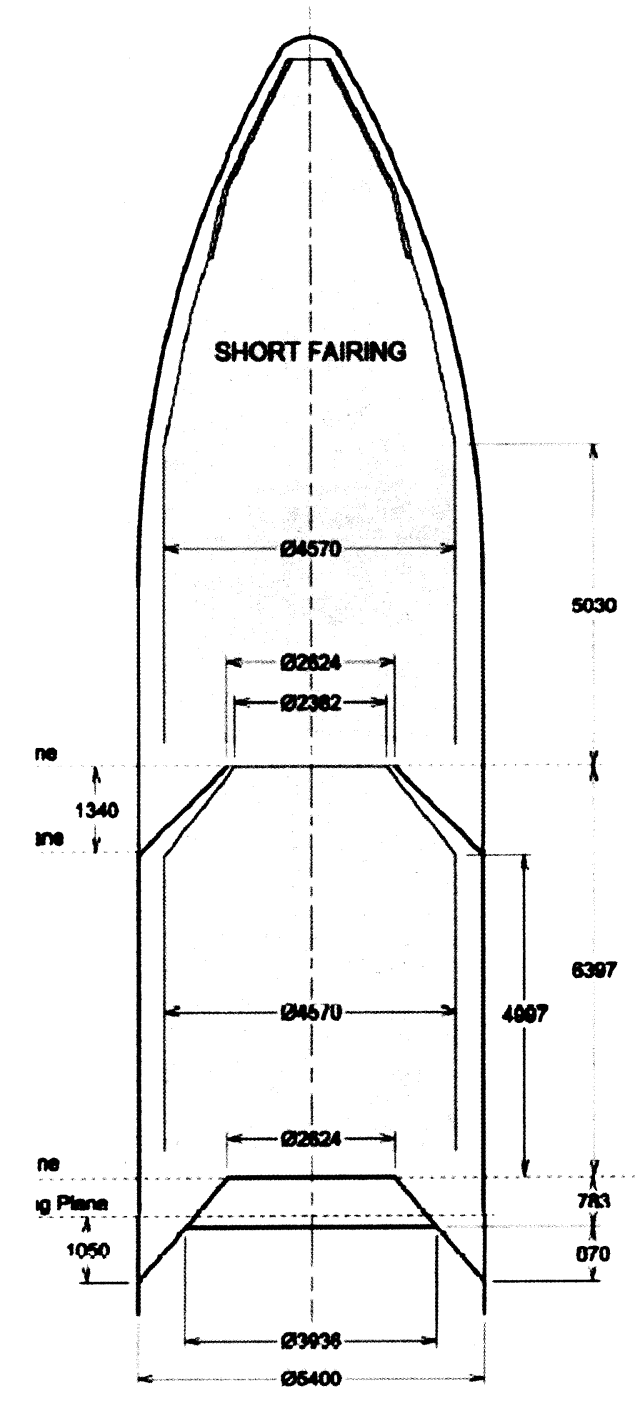

Ariane V

Long SPELTRA

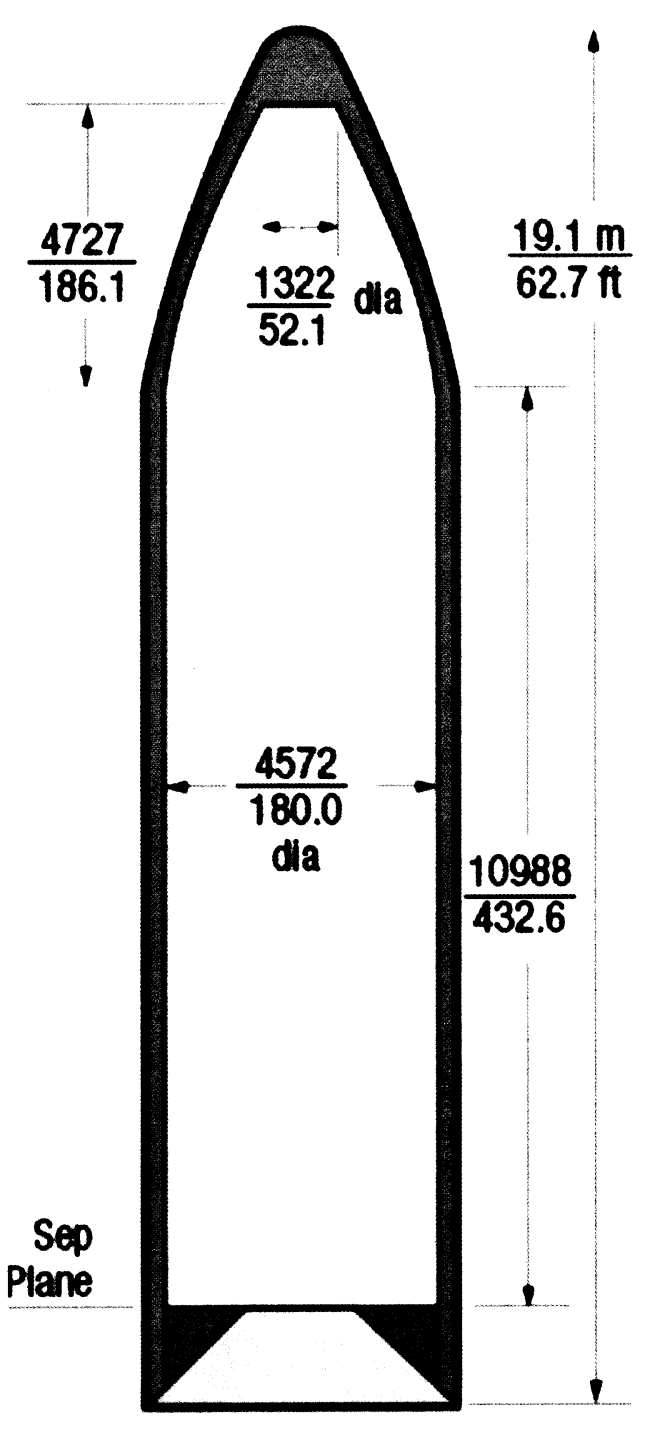

\author{
Delta IV heavy \\ Composite Fairing
}


Conceptual View of Possible Stowed Configuration of Telescope and Occulter (Courtesy of NGC)

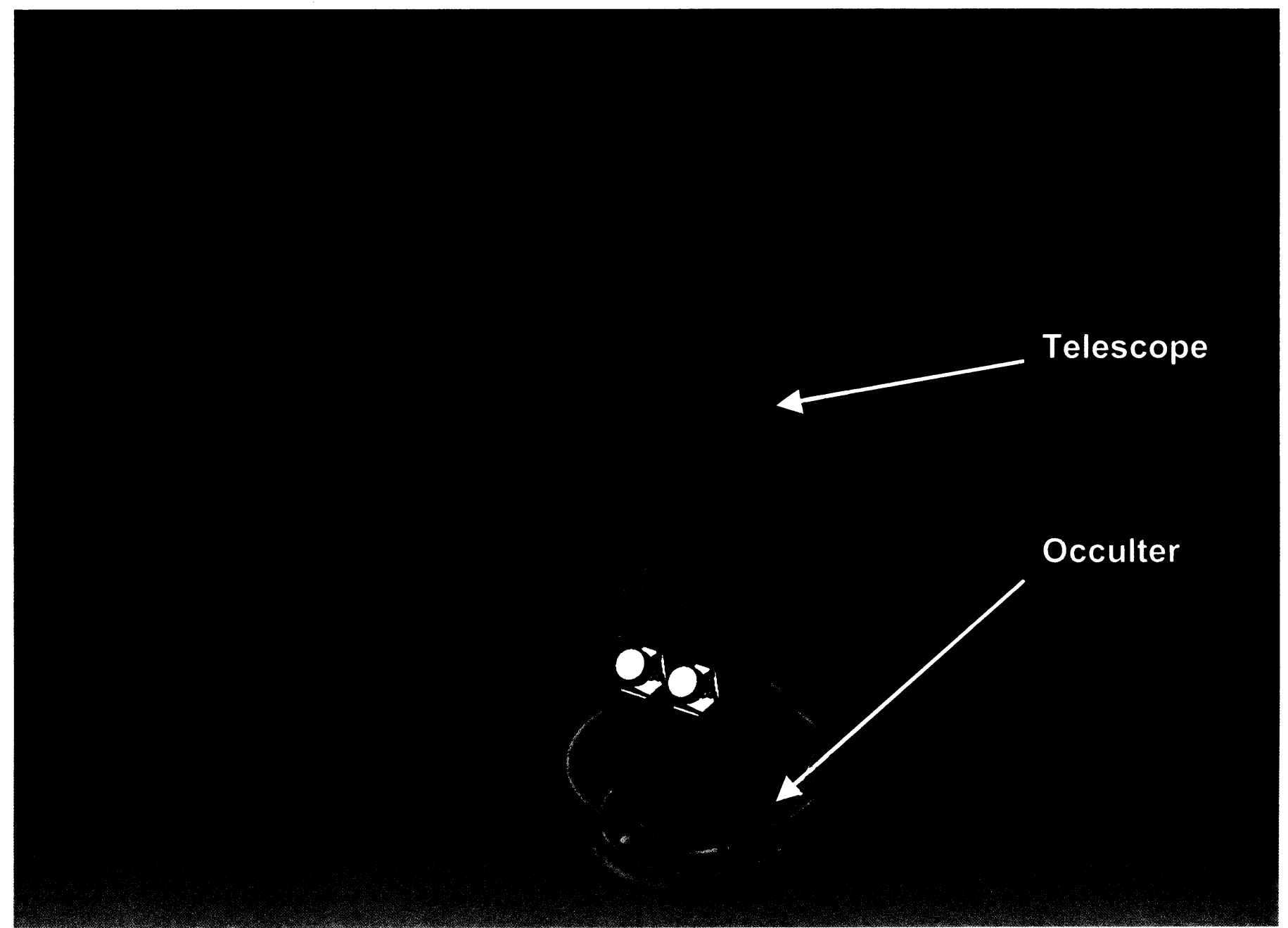




\section{Occulter SC Bus}

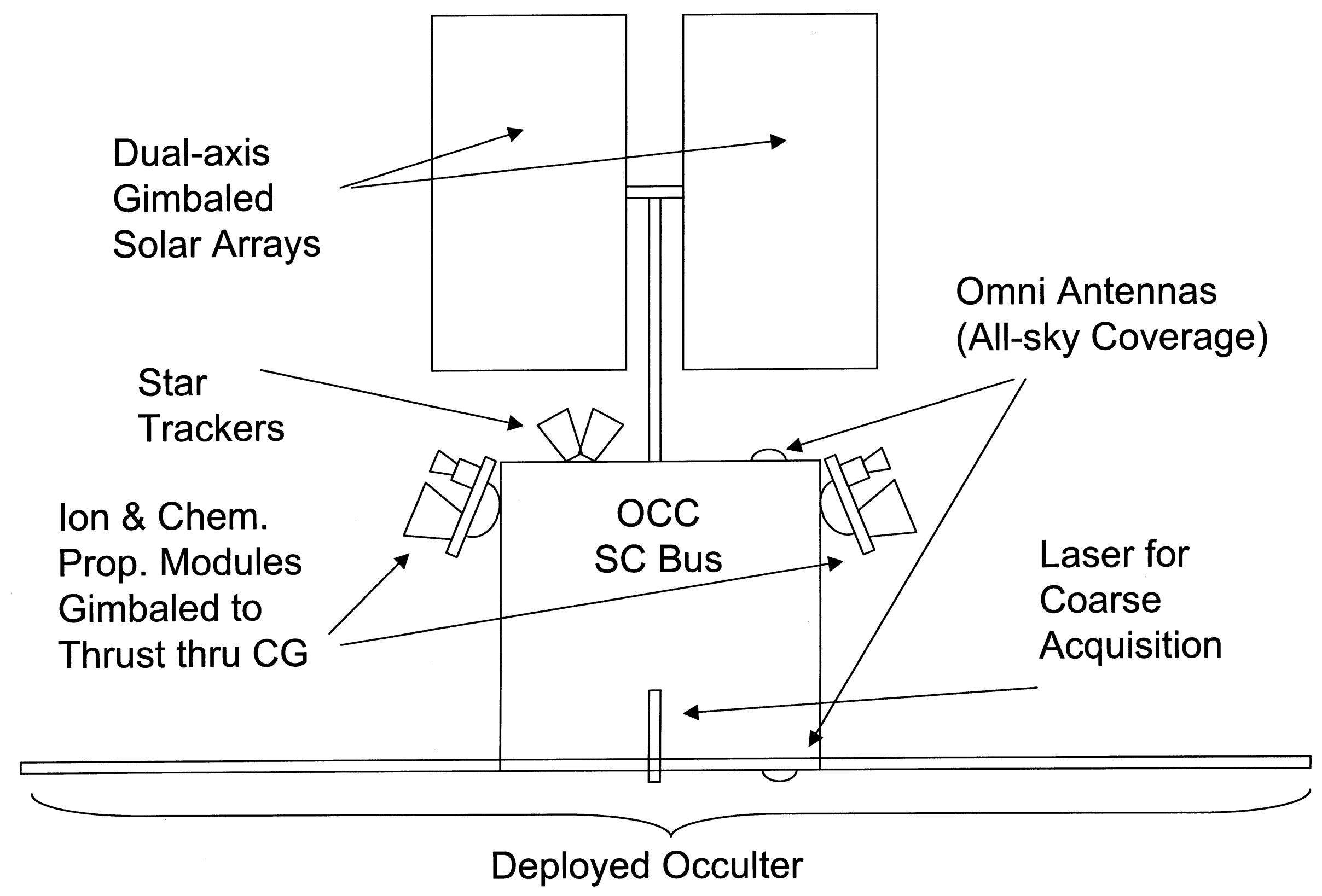




\section{Occulter - SC Bus Interface}

- For current definition, the occulter shade (with its deployment mechanisms and other required subsystems) is considered to be the payload of the SC bus with the following major interfaces

- Mechanical support for launch and operations

- Power to occulter for deployment motors, heaters, etc

- Bi-directional data for deployment control, thermal control, etc

- Reaction of occulter shade to thrust induced vibrations needs to be studied 


\section{Occulter SC Bus Functions}

- Structural

- Normal support functions, probably composite for low mass

- Maybe be oversized if required to support telescope during launch

- May include vibration isolation features

- Mechanical

- Deploy and gimbal solar arrays

- Possibly Gimbal thrusters

- Thermal: Normal heaters and thermocouples

- Propulsion

- Ion Propulsion System for sle maneuvers

- Chemical propulsion (monoprop looks adequate) for station keeping during science observations

- Attitude Control

- Star Trackers, Gyros, Reaction Wheels

- Safe hold sensors (coarse sun sensors) 


\section{Occulter SC Bus Functions (cont.)}

- Power

- Will need to generate $\sim 10 \mathrm{~kW}$ for ion engines

- No major challenges - Occulter should be near-constant sunlight at $\sim 99 \%$ level of intensity at Earth.

- Communications

- Transponder for tracking data to guide during slew maneuvers

- Pulsed laser transmitter to provide telescope with signal for coarse acquisition

- Probably patch antenna for communications with telescope

- S band Omni antennas for low data rate communications with Earth

- Command \& Data Handling (C\&DH)

- Normal functions of command processing and collecting health \& safety data

- Could run sophisticated orbit dynamics model to support maneuvers and station keeping 


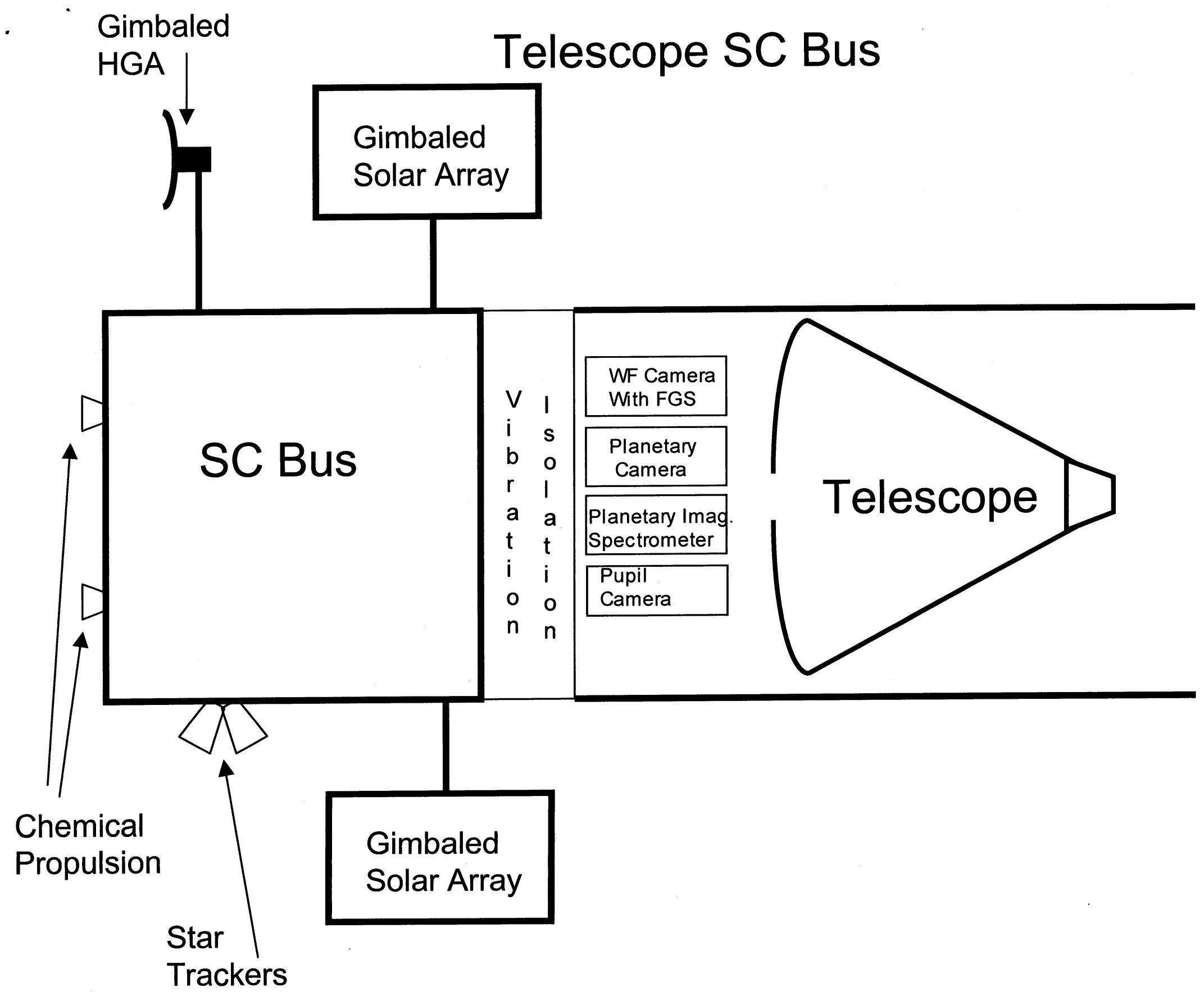




\section{Telescope - SC Bus Interface}

- For current definition, the telescope (with its instruments and associated electronics) is considered to be the payload of the SC bus with the following major interfaces

- Mechanical support for launch and operations

- Power

- Bi-directional data for instrument commands, science data return, health \& safety data

- Telescope provides following info for SC bus:

- FGS via WF camera

- Coarse acquisition of occulter via detecting laser light

- Tracking of occulter via diffracted IR light form target star 


\section{Telescope SC Bus Functions}

- Structural

- Normal support functions, probably composite for low mass

- Will include vibration isolation features

- Mechanical

- Deploy and gimbal solar arrays and High Gain Antenna (HGS)

- Thermal: Normal heaters and thermocouples

- Propulsion

- Chemical propulsion (mono-prop looks adequate) for L2 orbit insertion and orbit maintenance during mission

- Attitude Control

- Star Trackers, Gyros, Reaction Wheels

- Safe hold sensors (coarse sun sensors)

- Telescope Functions

- WF Camera for Fine Guidance System (FGS)

- WF/Planetary Camera for coarse acquisition of occulter by observing light from laser

- Pupil camera for providing fine position knowledge to occulter by observing target star IR light from that is diffracted around occulter 


\section{Telescope SC Bus Functions (cont.)}

- Power

- Will need to generate a few $\mathrm{kW}$ for all requirements

- No major challenges - Telescope should be in near-constant sunlight at $\sim 99 \%$ level of intensity at Earth.

- Communications

- Transponder for tracking data for orbit maintenance

- Gimbaled Ka Band HGA to return science data to earth

- Expected to use DSN antennas, but dedicated antenna is an option

- Can potentially have continuous DSN contact, but expensive

- KA band can deliver up to $1 \mathrm{Gbps}$ (expected to be driven by WF camera)

- Probably patch antenna for communications with occulter

- S band Omni antennas for low data rate communications with Earth

- Command \& Data Handling (C\&DH)

- Normal functions of command processing and collecting health \& safety data 


\section{Orbital Express Mission}

- DARPA Robotic Mission from

ASTRO \& NEXTSAT 3/8/06 thru 7/21/07

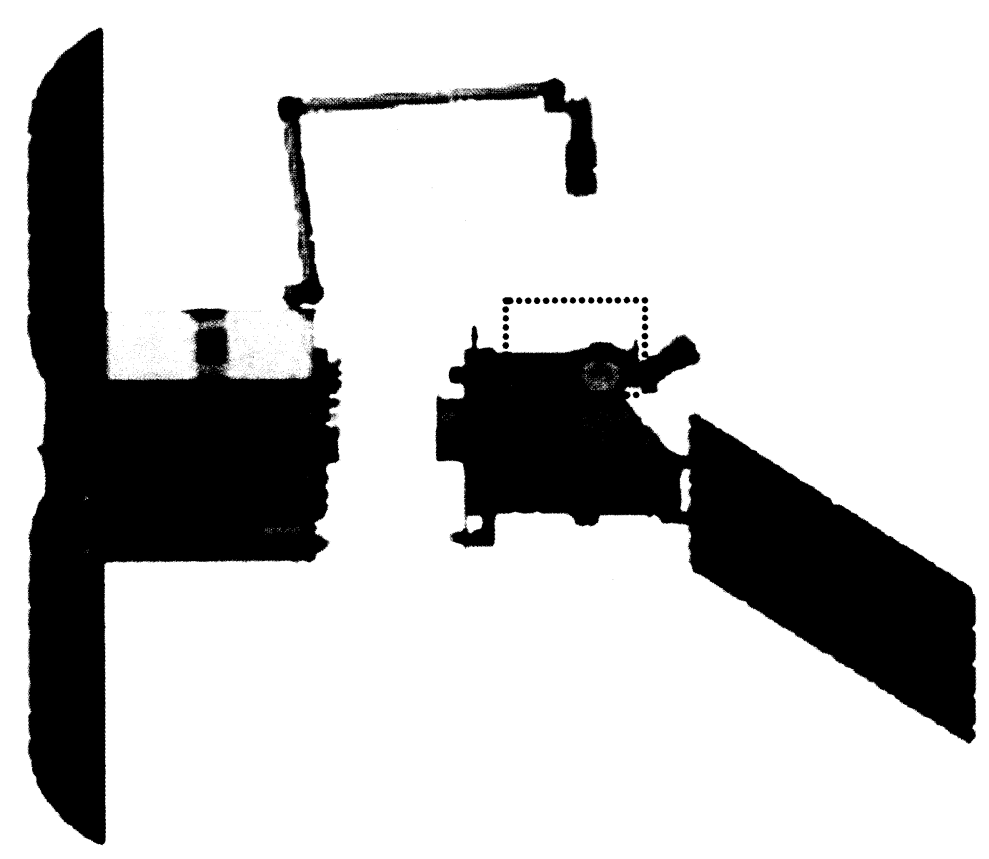

- Successfully Demonstrated

- Rendezvous

- Proximity Operations

- Station Keeping

- Capture \&Docking

- Fluid (Hydrazine) Transfer

- ORU (Orbit Replaceable

- Unit) Transfer

- Relevance: Provides high TRL Technology for servicing TPF-O 


\section{TPF-O SC-Bus Conclusions}

- Extensive Heritage

- HST: Serviceable SC Bus for UV/VIS/NIR ST

- SOHO in L1 Halo Orbit: L2-like Orbit dynamics

- WMAP in L2 Lissajous Orbit: L2 Environment

- JWST: ST in orbit being considered for TPF-O

- OE: Robotic servicing demonstartion

- Available technology can satisfy all known TPF-O SC Bus requirements

- But too early in mission design process to defined detailed requirements, design, masses, costs

- Challenges

- Large Delta V for Occulter Transfer Maneuvers

- Occulter coarse acquisition and tracking

- Occulter Settling time for Sci. Obs

- Design for compactness, low mass, low cost, robot servicing 